ORIGINAL ARTICLE

\title{
Understanding the Muscle Activity Pattern of the Hip Flexors during Straight Leg Raising in Healthy Subjects
}

\author{
Masahiro Yamane, PT, MS a Mitsuhiro Aoki, MD, PhD ${ }^{\text {b }}$ \\ Yuji Sasaki, PT, MS ${ }^{\mathbf{b}}$ and Hayato Kawaji, PT, MS ${ }^{\text {a }}$
}

\begin{abstract}
Objective: The aim of this study was to elucidate the activities of the hip flexor muscles during straight leg raising (SLR) in healthy subjects. We also investigated the activities of these muscles during SLR with deep flexion, abduction, and external rotation. Methods: The ten dominant right legs of ten male volunteers were analyzed in this study. Twelve SLR motion tasks were performed; these comprised combinations of hip flexion at $30^{\circ}, 45^{\circ}$, and $60^{\circ}$; abduction at $0^{\circ}$ and $20^{\circ}$; and external rotation at $0^{\circ}$ and $30^{\circ}$. The activities of the psoas major (PM) and iliacus (IL) were measured using fine-wire electrodes, whereas the activities of the rectus femoris, sartorius, adductor longus, and tensor fasciae latae muscles were measured using surface electrodes. The percentage of the maximal voluntary isometric muscle contraction (\%MVC) during SLR was calculated for each muscle and used for data analyses. The Friedman test and the Wilcoxon signed-rank test were performed for statistical analyses. The significance level was set at $\mathrm{P}<0.05$. Results: The $\% \mathrm{MVCs}$ for the PM and IL at $60^{\circ}$ flexion were significantly larger than those at $30^{\circ}$ or $45^{\circ}$ flexion. Moreover, for a constant hip flexion, the \%MVC values for the PM and IL showed no significant changes when hip abduction and external rotation were added. For the other muscles, the \%MVC values showed no significant change with increasing hip flexion with or without added abduction and external rotation. Conclusion: Our findings suggest that subjects who perform SLR of up to $60^{\circ}$ mainly activate the PM and IL at larger hip flexion angles, whereas the other muscles included in the analysis do not contribute greatly to increased flexion angles during SLR.
\end{abstract}

Key Words: biomechanics; electromyography; flexor muscles; hip joint; straight leg raising

\section{INTRODUCTION}

Straight leg raising (SLR) is a common method used for performing functional tests of the hip and pelvis. ${ }^{1-5)}$ This is used for athletes and patients to facilitate muscle activity of the trunk and hip flexors and to improve strength and coordination. ${ }^{6-9)}$

The hip flexor muscles comprise the psoas major (PM), iliacus (IL), rectus femoris (RF), sartorius (SAR), adductor longus (AL), and tensor fasciae latae (TFL). Previous studies have reported the muscle activity of the PM and IL during SLR $;^{7-10)}$ however, few studies have measured the muscle activities of other hip flexor muscles together with those of the PM and IL. ${ }^{7,10)} \mathrm{Hu}$ et al. measured the muscle activities of the
PM, IL, RF, and AL during SLR in which subjects raised the heel of one leg $20 \mathrm{~cm}$ above the table. ${ }^{10)}$ Thereafter, they reported bilateral muscle activities of the PM without inducing contralateral activity of the RF, SAR, AL, and TFL. Using a musculoskeletal model, Lewis et al. reported that the PM and IL showed greater activities than other hip flexor muscles; however, when the PM and IL activities were weakened in this model, the RF, SAR, AL, and TFL showed increased muscle activities. ${ }^{11)}$ This report also measured muscle activities during SLR with hip flexion of $0^{\circ}$ to $30^{\circ}$, with no deep hip flexion of $\geq 60^{\circ}$. Furthermore, they did not measure the muscle activities of the surrounding hip flexor muscles (i.e., the RF, SAR, AL, and TFL) simultaneously. Consequently, it is important to measure the muscle activities of all hip

Received: December 18, 2018, Accepted: February 6, 2019, Published online: February 16, 2019

${ }^{a}$ Department of Rehabilitation, Health Sciences University of Hokkaido Hospital, Sapporo, Japan

${ }^{b}$ Department of Physical Therapy, School of Rehabilitation Sciences, Health Sciences University of Hokkaido, Tobetsu, Japan

Correspondence: Masahiro Yamane, Department of Rehabilitation, Health Sciences University of Hokkaido Hospital, 2-5 Ainosato,

Kita-ku, Sapporo, Hokkaido 002-8072, Japan, E-mail: m-yamane@hoku-iryo-u.ac.jp

Copyright (C) 2019 The Japanese Association of Rehabilitation Medicine 


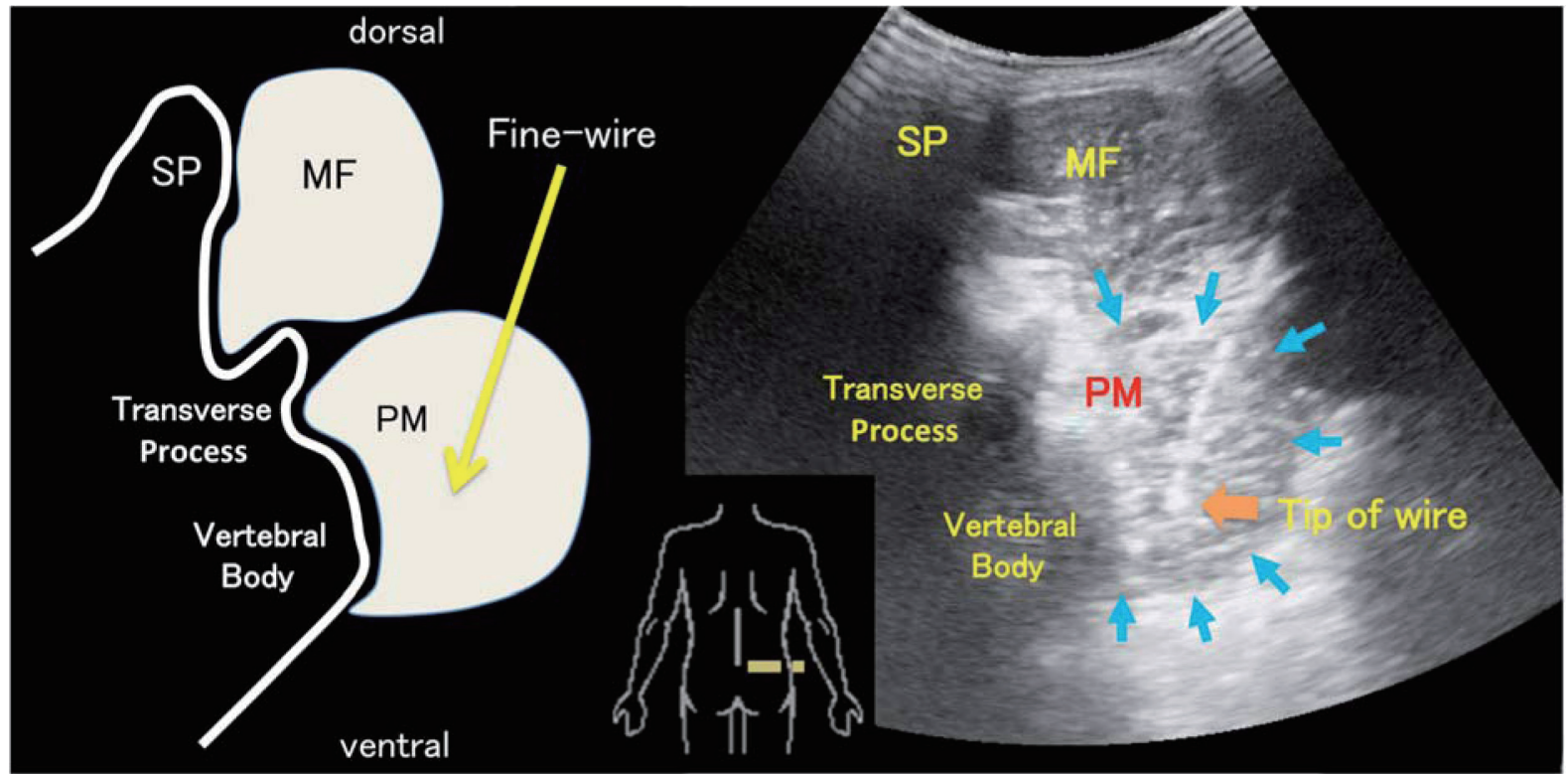

Fig. 1. Ultrasonography image of the wire insertion into the psoas major muscle. The image on the right is the ultrasonography image, and the one on the left is a line drawing based on the ultrasonography image. Small arrows (right image) indicate the outline of the psoas major muscle. The large arrow (right image) indicates the tip of the wire. The long arrow (left image) indicates the fine-wire electrode. MF, multifidus; SP, spinous process.

flexors during SLR with a wide range of motion to facilitate an understanding regarding the mechanism of SLR.

The objective of the current study was to elucidate the activities of the hip flexor muscles during SLR in healthy subjects. We also investigated the activities of these muscles during SLR with deep flexion, abduction, and external rotation. Thereafter, we discussed the role of the various hip flexors in SLR.

\section{MATERIALS AND METHODS}

\section{Participants}

The ten dominant right legs of ten male volunteers were analyzed in this study. The mean age, height, weight, and body mass index of the subjects were $21.1 \pm 1.0$ years, 174.7 $\pm 5.0 \mathrm{~cm}, 66.8 \pm 6.5 \mathrm{~kg}$, and $21.9 \pm 1.5 \mathrm{~kg} / \mathrm{m}^{2}$, respectively. Based on previous studies, ten participants were enrolled. Participants were excluded if they had a history of lumbar, pelvis, hip, or lower extremity disease; neurological deficit; a physical condition with a passive SLR angle of $<60^{\circ}$; compensatory rotation and lordosis of the lumbar spine; or rotation of the pelvis during active SLR. Before study initiation, written informed consent was obtained from all subjects. All the procedures were approved by the Ethical Committee of the Health Sciences University of Hokkaido (approval num- ber 16R039041).

\section{Electromyography}

Pairs of fine-wire electrodes (urethane coated, $50 \mu \mathrm{m}$ diameter $\times 250 \mathrm{~mm}$ length, stainless-steel wire, $1 \mathrm{~mm}$ of urethane removed from the tips; Unique Medical Co., Tokyo, Japan, threaded into a hypodermic needle $[0.72 \mathrm{~mm}$ diameter $\times 100 \mathrm{~mm}$ length for PM and $0.72 \mathrm{~mm}$ diameter $\times 70 \mathrm{~mm}$ length for IL], bent back to form 5-mm hooks) were inserted into the right PM and IL. These paired electrodes were used as bipolar electrodes. Surface electrodes (cordless active electrode: the width, length, and interelectrode distance were $1 \mathrm{~mm}, 10 \mathrm{~mm}$, and $10 \mathrm{~mm}$, respectively; Nihon Koden Ltd, Tokyo, Japan) were placed on the right RF, SAR, AL, and TFL. Before inserting the wire or placing the surface electrodes, the skin was scrubbed and wiped with $70 \%$ alcohol. Based on previous studies, ${ }^{8,12-14)}$ under ultrasonographic guidance (using the convex probe of an HS1 ultrasound system, Konica Minolta, Osaka, Japan), fine-wire electrodes for the PM were inserted to a depth of 7-9 $\mathrm{cm}$ from the skin via a route $7 \mathrm{~cm}$ lateral to the spinous process between the L3-L4 transverse process at an angle of $20^{\circ}$ to the sagittal plane. The tip of the wire was placed in the muscle $2 \mathrm{~cm}$ away from the anterior fascia (Fig. 1). For the IL, fine-wire electrodes were inserted in the center of the muscle belly 


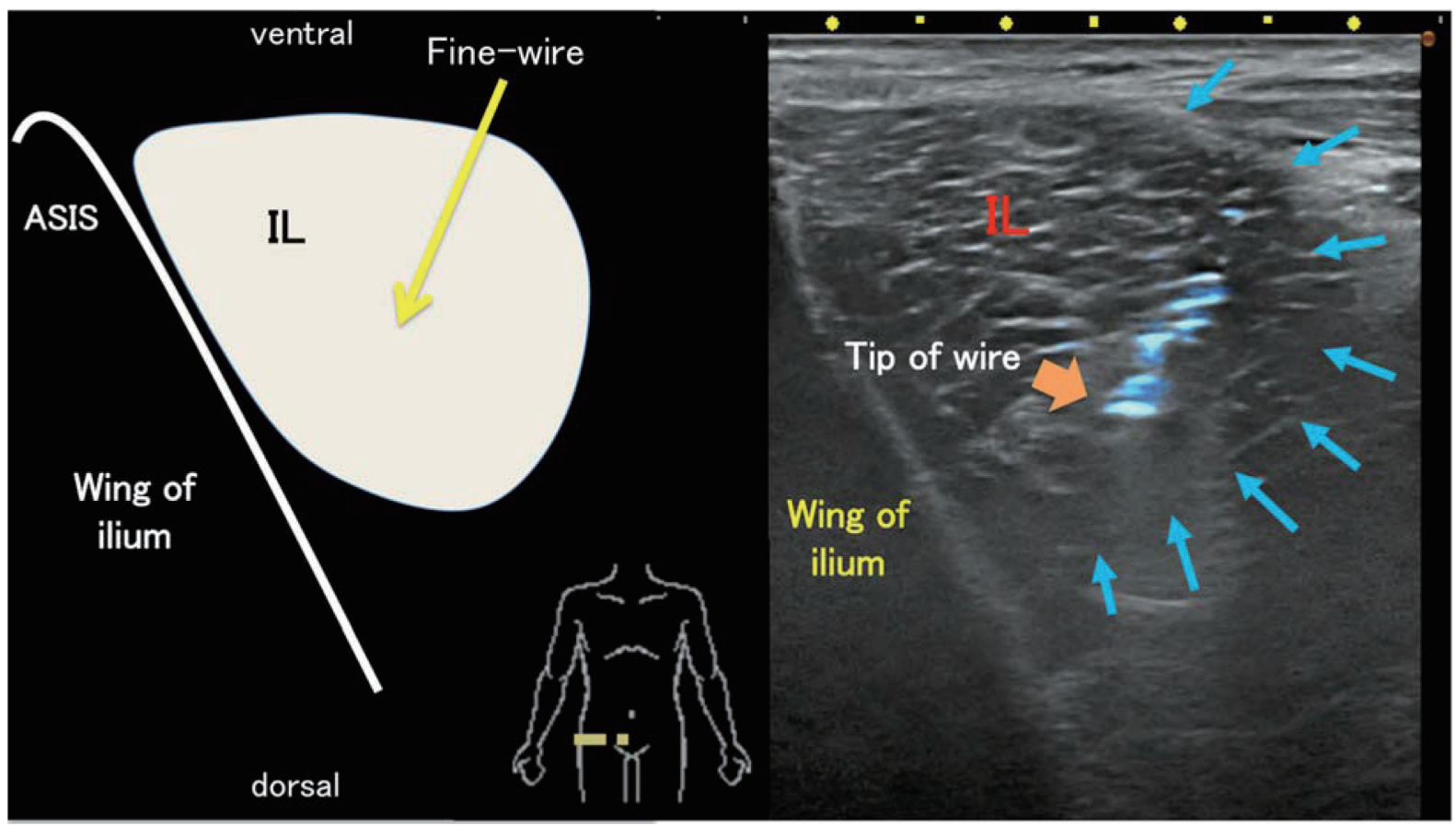

Fig. 2. Ultrasonography image of wire insertion into the iliacus muscle. The image on the right is the ultrasonography image, and the one on the left is a line drawing based on the ultrasonography image. Small arrows (right image) indicate the outline of the iliacus muscle. The large arrow (right image) indicates the tip of the wire. The long arrow (left image) indicates the fine-wire electrode.

from a location $2 \mathrm{~cm}$ medial to the anterior superior iliac spine (ASIS) and $1 \mathrm{~cm}$ distal to the inguinal ligament (Fig. 2). After the fine-wire electrodes were inserted into the PM and IL, ultrasonographic examination was used to confirm correct wire insertion into these muscles by observing muscle contraction during submaximal twitch stimuli (nerve stimulator, TS-260, Keisei Medical Co, Tokyo, Japan). The fine-wire electrodes were inserted into the PM and IL by an experienced orthopedic surgeon. Based on Perotto's method, the surface electrodes were placed on the midpoint between the superior border of the patella and ASIS for the RF, 5-6 cm distal from the ASIS along the line between the ASIS and the medial condyle of the tibia for the SAR, 5-6 cm from the pubic tubercle for the AL, and 2-3 cm anterior to the greater trochanter for the TFL. ${ }^{15)}$ Surface electrodes were placed on each muscle belly, parallel to the muscle fiber orientation.

Recordings from the electrodes were acquired using an RMT-1000 polygraph system (Nihon Koden, Tokyo) for the fine-wire electrodes and a Web-1000 (Nihon Koden, Tokyo) for the surface electrodes. All electromyography (EMG) data were synchronized using the RMT-1000. Once the electrodes were in position, the participants performed active SLR three times, and we confirmed that the EMG data had been recorded successfully with minimum noise (Fig. 3). EMG signals were sampled at $1000 \mathrm{~Hz}$ and stored in a personal computer.

\section{Measurement of Maximal Voluntary Contrac- tion}

Based on the methods of manual muscle testing, the isometric maximal voluntary contraction (MVC) for each muscle was measured against manual resistance for $5 \mathrm{~s}$ in all patients; three trials were performed. ${ }^{16)}$ MVC values for the PM, IL, RF, and SAR were measured with the subject in the sitting position, and those of the AL and TFL were recorded with the subject lying on their side. Prior to the measurement of MVC for each muscle, subjects were asked to perform three trials, with a 1 -min rest period between trials.

\section{Procedure}

Twelve SLR motion tasks that comprised a combination of hip flexion at $30^{\circ}, 45^{\circ}$, and $60^{\circ}$; abduction at $0^{\circ}$ or $20^{\circ}$; and external rotation at $0^{\circ}$ or $30^{\circ}$ were utilized. Motion tasks were performed in a random order to prevent sequence effects. Participants were placed in the supine position on a bed containing a window through which passed the wires 


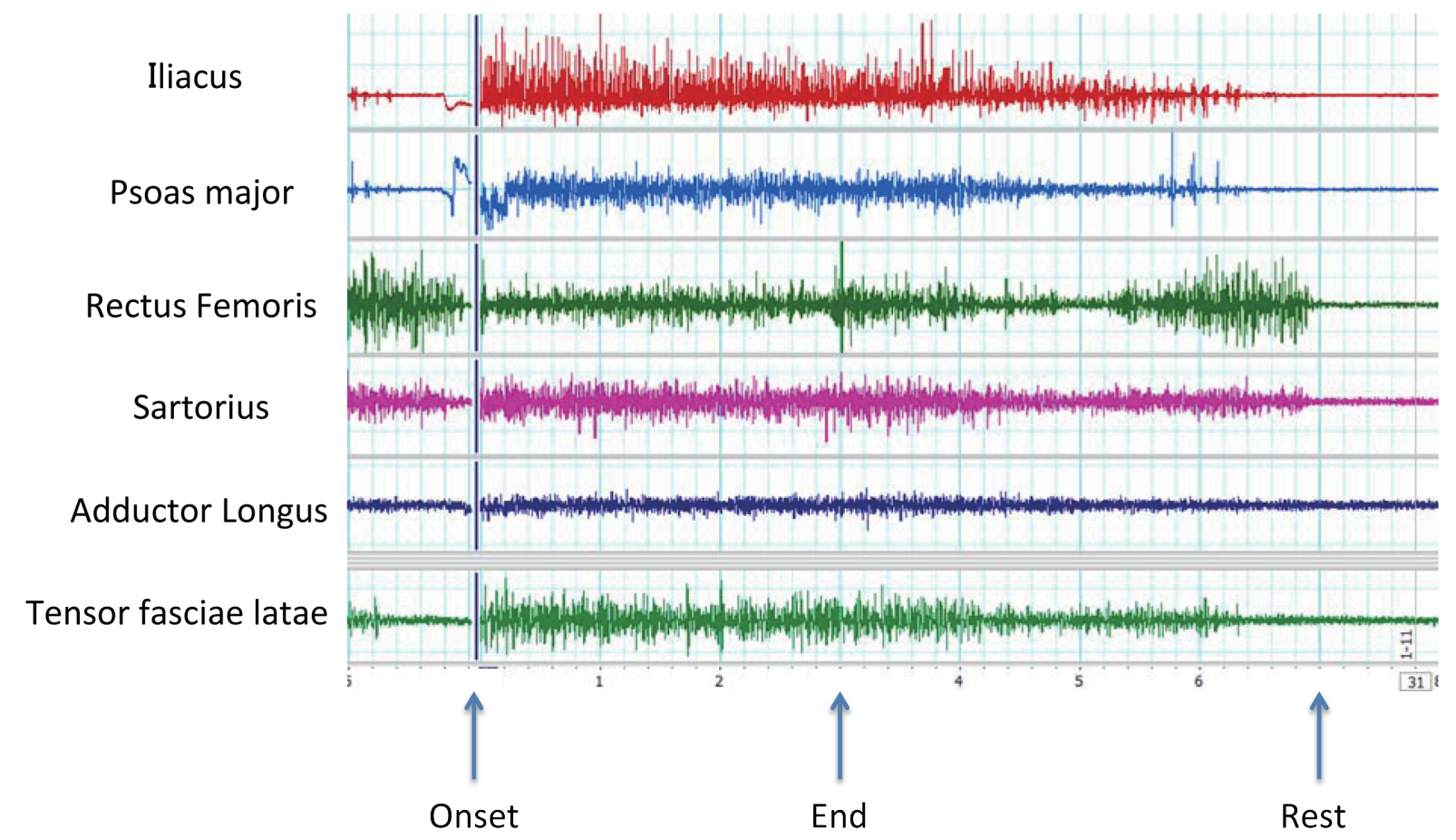

Fig. 3. Electromyographic activity of each muscle during a straight leg raising motion. Electrical activities of each muscle persist from the onset of recording (Onset arrow) to the end of the recording (End arrow) during SLR motion. Thereafter, the activities fade away to those at baseline (Rest arrow).

that were inserted into the participant's back. This setup minimized pressure artifacts in the electrical recording of SLR activity. The initial position of the participants was hip flexion/extension neutral, adduction/abduction neutral, internal/external rotation neutral, full knee extension, and ankle dorsiflexion of $0^{\circ}$. To prevent pelvic rotation, the left thigh was fixed to the bed using a belt. Initially, each participant was asked to position his right hip at a certain degree of abduction or external rotation on the bed. Next, the participant was asked to elevate the right leg to one of the flexion angles of the SLR positions by an assistant examiner using a goniometer. Thereafter, a sling of the Redcord trainer (Redcord AS, Arendal, Norway) held the ankle in the designated SLR position. When EMG was performed, the sling holding the leg in the designated flexion angle was removed, and the subject maintained that SLR flexion angle, keeping hip abduction and external rotation constant with knee full extension and ankle dorsiflexion at $0^{\circ}$. EMG with the leg in the specified SLR position was performed for $3 \mathrm{~s}$, and the readings of three trials were obtained for each of the 12 positions. Hip angle setting was performed for every trial. To minimize fatigue, the participants were allowed a 1-min rest period between subsequent trials. The range of hip joint flexion and abduction motion was measured according to the standard of the Japan Orthopedic Association using a goniometer. Based on Kumamoto's method, the angle between the vertical and the line connecting the center of the heel and the interval between the first and second toe was defined as the external/internal rotation ${ }^{17)}$ (Fig. 4).

\section{Data Analysis}

Wire and surface EMG data were collected and analyzed using Labchart version 7 (AD Instruments). EMG data were band-pass filtered in the range $20-500 \mathrm{~Hz}$ for both surface and wire electrodes to remove movement artifacts (i.e., a fourth-order Butterworth filter). A 0.5-s period of EMG data during stable SLR monitoring was selected from the $3 \mathrm{~s}$ of data and used for analysis (Fig. 3). After the EMG signals were full-wave rectified, the root mean square (RMS) was calculated. As part of the previous MVC measurements, the mean RMS value for each muscle was calculated from the three trials. The mean value for each muscle during SLR was normalized using the mean RMS value during MVC for each muscle and expressed as \%MVC. 

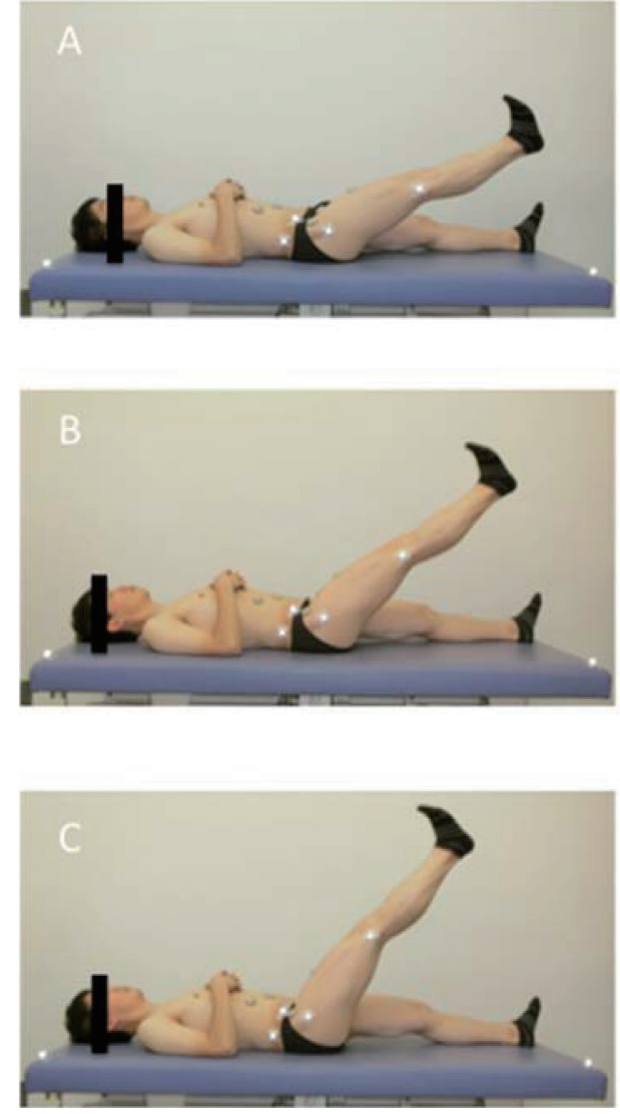
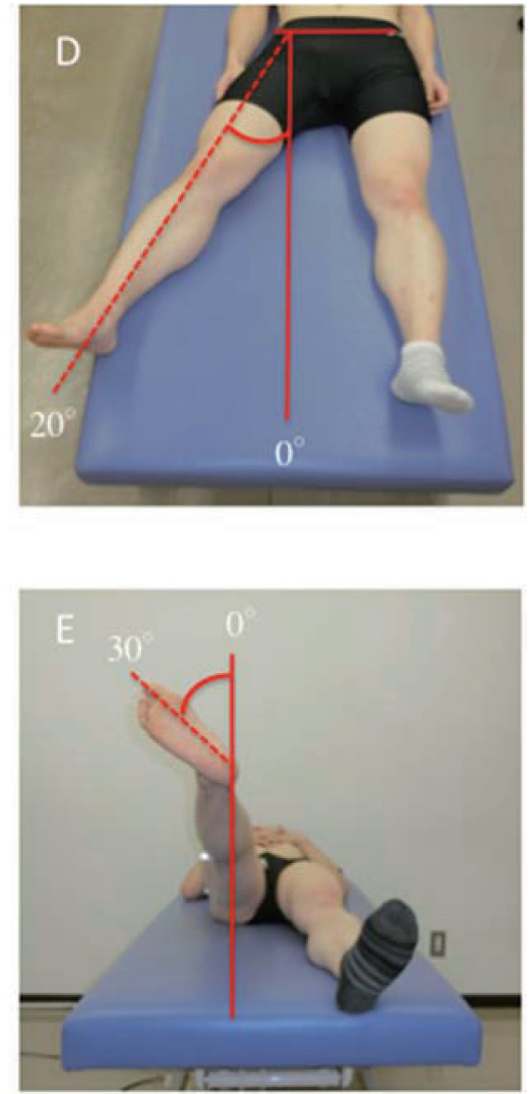

Fig. 4. Hip positions during straight leg raising motions. In the lateral view, we define the hip flexion angle in the supine position as the angle between the longitudinal line of the femur and the trunk axis. (A) Hip flexion $30^{\circ}$, (B) hip flexion $45^{\circ}$, and (C) hip flexion $60^{\circ}$. In the frontal view, we defined the hip abduction angle as the angle between the longitudinal line of the femur and the line connecting the bilateral anterior superior iliac spine. (D) Hip abduction $0^{\circ}$ and $20^{\circ}$. On the axial view, we defined the angle between the vertical and the line connecting the center of the heel and the interval between the first and second toe as the external rotation angle. (E) Hip external rotation $0^{\circ}$ and $30^{\circ}$.

\section{Statistical Analysis}

Statistical analysis was performed using $\mathrm{R}$ 2.8.1. The data were analyzed for the four distinct conditions of hip abduction and external rotation. The \%MVC data for each muscle were examined using the Shapiro-Wilk test to determine whether the distribution was normal. Thereafter, we performed Friedman nonparametric testing to analyze each muscle during hip flexion in SLR. Post-hoc testing was performed using the Wilcoxon signed-rank test with Bonferroni correction. To assess the effects of abduction and/or external rotation, we performed Friedman nonparametric testing to analyze each muscle during SLR at a fixed hip flexion. The significance level was set at $\mathrm{P}<0.05$.

\section{RESULTS}

\section{Participant Characteristics}

No subjects fulfilled any exclusion criteria, and all completed the experiment. We did not observe any physical or mental adverse event during or after the experiment.

\section{Muscle Activities of the Hip Flexors}

The \%MVC values of the recorded muscles for each of the 12 SLR position are shown in Table 1. For the PM, the $\% \mathrm{MVC}$ at $60^{\circ}$ flexion was significantly larger than those at $30^{\circ}$ and $45^{\circ}$ flexion for an external rotation of $0^{\circ}$ and abduction angles of $0^{\circ}$ and $20^{\circ}$ (Fig. 5A, 5C). For an abduction angle of $20^{\circ}$ with $30^{\circ}$ of external rotation, the $\% \mathrm{MVC}$ for the PM at $60^{\circ}$ flexion was significantly larger than that at 


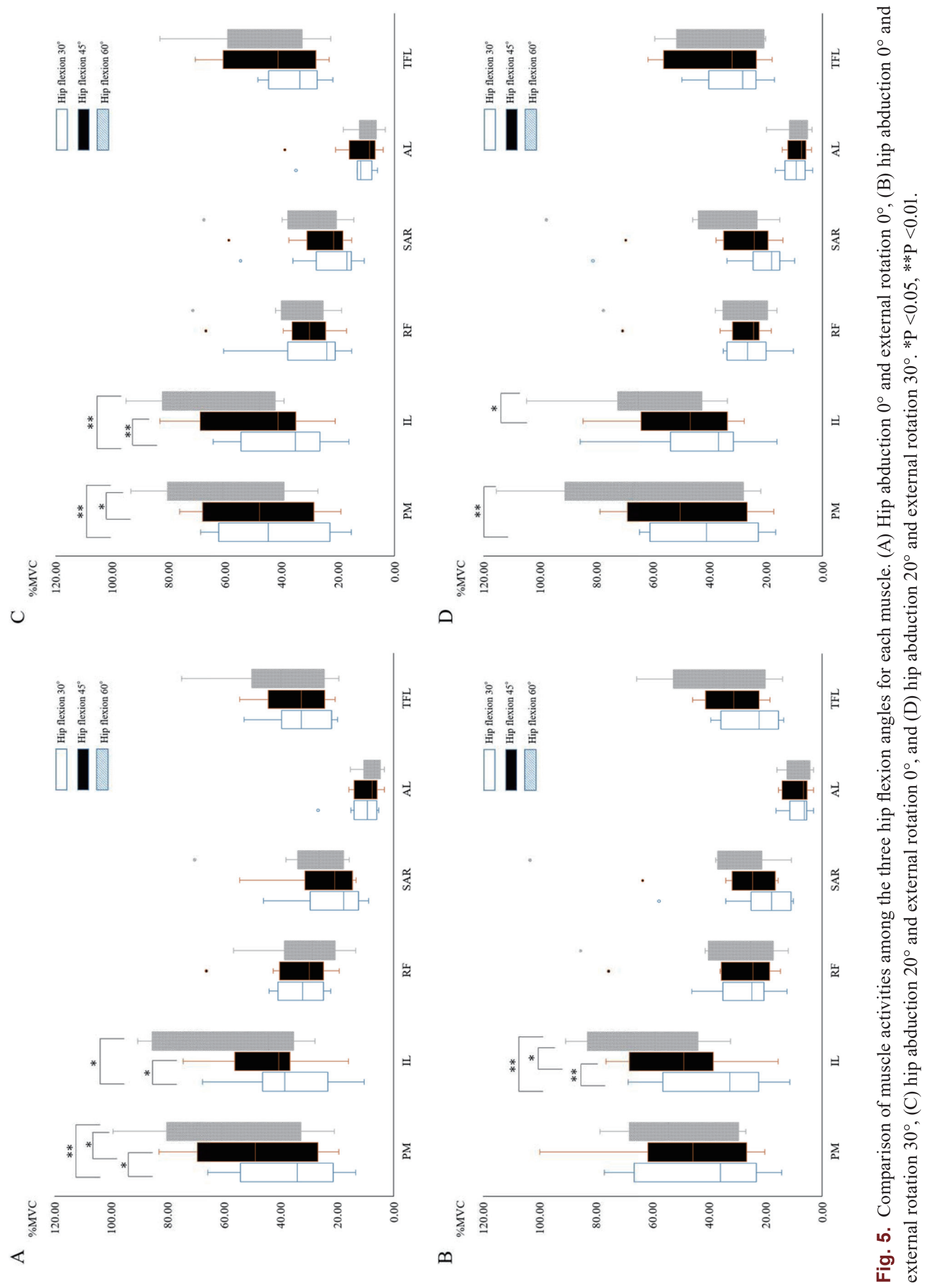




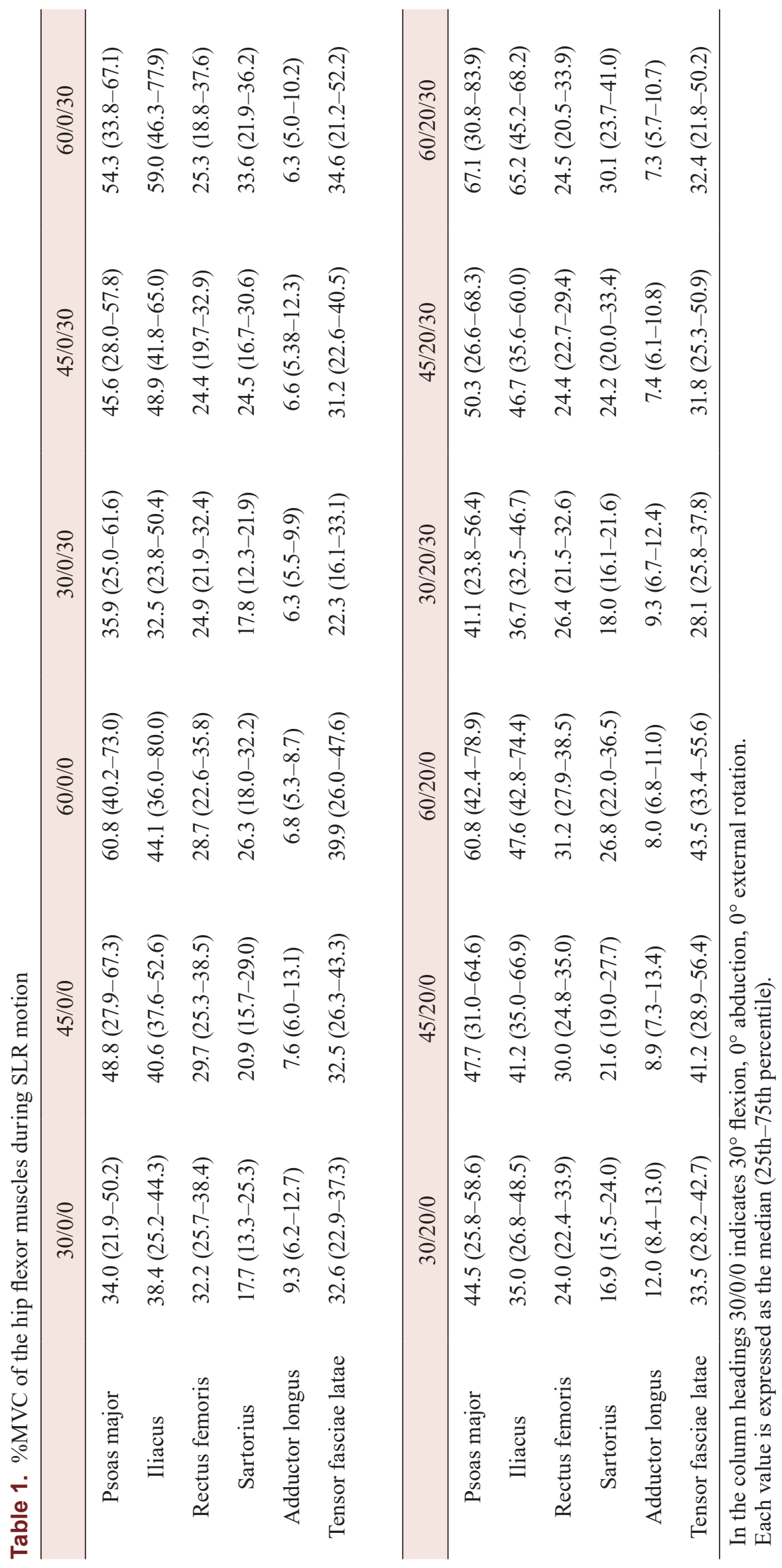


$30^{\circ}$ flexion (Fig. 5D). For a constant hip flexion, the PM \%MVC values showed no significant changes when hip abduction and external rotation were added. For the IL, the $\% \mathrm{MVC}$ values were significantly larger at $60^{\circ}$ flexion than those at $30^{\circ}$ or $45^{\circ}$ flexion, and hip abduction and external rotation did not influence these differences (Fig. 5A-D). For a constant hip flexion, the IL \%MVC values showed no significant changes when hip abduction and external rotation was added. To summarize, the PM and IL showed greater activity when the hip flexion angle increased. For the RF, SAR, AL, and TFL, the \%MVC values showed no significant change with increasing hip flexion or hip flexion with abduction and external rotation (Fig. 5A-D).

\section{DISCUSSION}

We measured the activities of the muscles that flex the hip during SLR. The PM and IL showed significantly greater muscle activity with increases in the hip flexion angle, whereas the activities of the other muscles did not show a significant increase. Moreover, for a constant hip flexion, all the muscles investigated showed no significant change in muscle activity during SLR when hip abduction and external rotation were added. To the best of our knowledge, the present study is the first to report the simultaneously measured electrical activities of all major hip flexor muscles during SLR and the associations with hip abduction and external rotation.

\section{Relationship of Muscle Activity with the Flex- ion Angle}

Our results showed that the activity of the PM and IL increased with increasing hip flexion; however, the other muscles did not show any changes in activity for all hip flexion positions. Yoshio et al. examined cadaveric specimens to show that the PM is a stabilizer of the femoral head at $15^{\circ}$ SLR and is estimated to produce hip flexion at $>45^{\circ}$ SLR. ${ }^{18)}$ Previous studies using fine-wire electrodes have reported that the PM is activated during deep hip flexion. ${ }^{8,9,19)}$ Andersson et al. reported that IL activity is greater with increased hip flexion. ${ }^{8)}$ Hirano et al. reported that the anterior fibers of the IL are activated more at initial hip flexion; further, the middle/posterior fibers of the IL, which run in the craniocaudal direction and merge with the PM, reportedly showed a similar working pattern to that of the PM. ${ }^{20)}$ Thus, the activity patterns of the PM and IL during SLR are similar.

Our results showed no significant increase in the activities of the RF, SAR, AL, or TFL at any SLR position. Similar to our findings, the results of the study by Jiroumaru et al. showed that, during hip flexion, the activity of the SAR and TFL significantly decreased because their muscle length shortened with increased hip flexion angle. Moreover, the activity of the RF did not change significantly, irrespective of the hip flexion angle. ${ }^{21)}$ Using a mathematical model, Ogaya et al. analyzed the flexion torque of the hip generated by the surrounding hip muscles. ${ }^{22)}$ Based on this report, the torque of the hip increased by the action of the iliopsoas during hip flexion greater than $30^{\circ}$; whereas, the peak torque of the RF was observed at a hip flexion of $20^{\circ}$. Blemker et al. reported that the moment arm of the PM and IL were large at hip flexions in the range $70^{\circ}-80^{\circ}{ }^{23)}$ In our study, the activities of the PM and IL were significantly larger at $60^{\circ}$ hip flexion than at $30^{\circ}$ or $45^{\circ}$. Consequently, we suggest that SLR at $60^{\circ}$ hip flexion produces a large flexion torque by the action of the PM and IL.

In the present study, for a constant hip flexion, there was no significant change in the activities of the PM and IL during SLR with the addition of abduction and/or external rotation. Based on the observation by Kendall et al., hip flexion with abduction and external rotation was appropriate for evaluating the strength of the PM because the lesser trochanter shifts anteriorly as a result of the external rotation of the hip, and the muscle fiber of the PM runs straight for this hip position. ${ }^{24)}$ Skyrme et al. reported that the PM worked for pure flexion at the neutral hip abduction/adduction position, and worked for flexion and external rotation in the hip abduction position. ${ }^{25)}$ However, our results showed no significant difference between $0^{\circ}$ and $20^{\circ}$ of hip abduction or between $0^{\circ}$ and $30^{\circ}$ of external rotation in terms of the \%MVC values of the PM and IL. Keagy et al. reported the contribution of the PM to hip rotation and abduction/adduction is small. ${ }^{26}$ ) Therefore, the PM and IL mainly work for hip flexion during SLR, whereas they contribute little to hip abduction and external rotation.

Our results showed no significant increases in the activities of the RF, SAR, AL, and TFL at any SLR position. Nonaka et al. measured the \%MVC of the RF, SAR, and AL during SLR with external rotation and reported that the $\% \mathrm{MVC}$ of these muscles showed no significant change with increases in external rotation. ${ }^{27)}$

Based on our findings and those of previous reports, the PM and IL mainly work as hip flexors at larger hip flexion angles, and the other muscles work constantly in SLR for all positions, including the addition of abduction and external rotation. Further studies at SLR $>60^{\circ}$ may help elucidate the selective activities of PM and IL. 


\section{Clinical Application}

The functional roles of the PM and IL are important for SLR because the activities of the PM and IL were significantly greater as the hip flexion increased. In contrast, the activity of the other muscles was limited and was not depended on the hip flexion. It is suggested, therefore, that subjects with full passive SLR motion who cannot perform active SLR up to $60^{\circ}$ have weakness in the PM and IL.

\section{Limitations}

The present study had three major limitations. First, subjects whose SLR was $<60^{\circ}$ were excluded. Tightness of the hamstrings, which connect the pelvis to the thigh, might have influenced hip flexion in SLR. Second, subjects performed SLR motions without limiting the anterior/posterior pelvic movement. We did not record muscle activities with hip motion alone. Third, we evaluated only young male subjects. Older or female subjects might show different results.

\section{CONCLUSIONS}

We measured the activities of the hip flexor muscles during SLR in 12 positions and revealed the patterns in which these muscles were activated. The PM and IL showed significantly larger activity as the hip flexion angle increased, and, for a constant hip flexion, there was no significant change in activity with abduction and external rotation. The other muscles showed no significant change in activity in any position. Our study suggested that subjects who perform SLR of up to $60^{\circ}$ may activate the PM and IL.

\section{ACKNOWLEDGMENTS}

We acknowledge Yoshida S for EMG data analysis and Iizawa T, Sugawara $\mathrm{K}$, and Honma $\mathrm{H}$ for data collection. We thank Kaneoka $\mathrm{K}$ and Takasaki $\mathrm{H}$ for suggestions and editing the manuscript.

\section{CONFLICTS OF INTEREST}

There are no conflicts of interest to declare.

\section{REFERENCES}

1. Mens JM, Vleeming A, Snijders CJ, Stam HJ, Ginai AZ: The active straight leg raising test and mobility of the pelvic joints. Eur Spine J 1999;8:468-473. PMID:10664304, DOI:10.1007/s005860050206
2. de Groot M, Pool-Goudzwaard AL, Spoor CW, Snijders CJ: The active straight leg raising test (ASLR) in pregnant women: differences in muscle activity and force between patients and healthy subjects. Man Ther 2008;13:68-74. PMID:17188924, DOI:10.1016/j. math.2006.08.006

3. Mens JM, Pool-Goudzwaard A: The transverse abdominal muscle is excessively active during active straight leg raising in pregnancy-related posterior pelvic girdle pain: an observational study. BMC Musculoskelet Disord 2017;18:372. PMID:28841825, DOI:10.1186/s12891017-1732-9

4. Park K, Ha S, Kim S, Park K, Kwon O, Oh J: Effects of the pelvic rotatory control method on abdominal muscle activity and the pelvic rotation during active straight leg raising. Man Ther 2013;18:220-224. PMID:23127994, DOI:10.1016/j.math.2012.10.004

5. Grey C: Movement: Functional Movement Systems: Screening, Assessment, Corrective Strategies. Lotus Publishers, Punjab, 2011.

6. Hu H, Meijer OG, Hodges PW, Bruijn SM, Strijers RL, Nanayakkara PW, van Royen BJ, Wu W, Xia C, van Dieën JH: Understanding the active straight leg raise (ASLR): an electromyographic study in healthy subjects. Man Ther 2012;17:531-537. PMID:22728211, DOI:10.1016/j.math.2012.05.010

7. Hu H, Meijer OG, van Dieën JH, Hodges PW, Bruijn SM, Strijers RL, Nanayakkara PW, van Royen BJ, Wu W, Xia C: Muscle activity during the active straight leg raise (ASLR), and the effects of a pelvic belt on the ASLR and on treadmill walking. J Biomech 2010;43:532-539. PMID:19883914, DOI:10.1016/j.jbiomech.2009.09.035

8. Andersson E, Oddsson L, Grundström H, Thorstensson A: The role of the psoas and iliacus muscles for stability and movement of the lumbar spine, pelvis and hip. Scand J Med Sci Sports 1995;5:10-16. PMID:7882121, DOI:10.1111/j.1600-0838.1995.tb00004.x

9. Juker D, McGILL ST, Kropf P, Steffen T: Quantitative intramuscular myoelectric activity of lumbar portions of psoas and the abdominal wall during a wide variety of tasks. Med Sci Sports Exerc 1998;30:301-310. PMID:9502361, DOI:10.1097/00005768-19980200000020 
10. Hu H, Meijer OG, van Dieën JH, Hodges PW, Bruijn SM, Strijers RL, Nanayakkara PW, van Royen BJ, $\mathrm{Wu} \mathrm{WH}, \mathrm{Xia} \mathrm{C}$ : Is the psoas a hip flexor in the active straight leg raise? Eur Spine J 2011;20:759-765. PMID:20625774, DOI:10.1007/s00586-010-1508-5

11. Lewis CL, Sahrmann SA, Moran DW: Effect of position and alteration in synergist muscle force contribution on hip forces when performing hip strengthening exercises. Clin Biomech (Bristol, Avon) 2009;24:35-42. PMID:19028000, DOI:10.1016/j.clinbiomech.2008.09.006

12. Andersson EA, Nilsson J, Thorstensson A: Intramuscular EMG from the hip flexor muscles during human locomotion. Acta Physiol Scand 1997;161:361-370. PMID:9401589, DOI:10.1046/j.1365201X.1997.00225.X

13. Park RJ, Tsao H, Cresswell AG, Hodges PW: Differential activity of regions of the psoas major and quadratus lumborum during submaximal isometric trunk efforts. J Orthop Res 2012;30:311-318. PMID:21800359, DOI:10.1002/jor.21499

14. Park RJ, Tsao H, Claus A, Cresswell AG, Hodges PW: Changes in regional activity of the psoas major and quadratus lumborum with voluntary trunk and hip tasks and different spinal curvatures in sitting. J Orthop Sports Phys Ther 2013;43:74-82. PMID:22960605, DOI:10.2519/jospt.2013.4292

15. Perotto OA, Edward FD, Lazzetti J, Morrison D: Anatomical Guide for the Electromyographer: The Limb and Trunk, 5th Edn. Charles C Thomas Ltd, Springfield, 2011:219-340.

16. Helen H, Dale A, Marybeth B: Daniels and Worthingham's Muscle Testing: Techniques of Manual Examination and Performance Testing, 7th Edn. Saunders, United States, 2002.

17. Kumamoto T, Ito T: The influence of vastus medialis muscle activity on different hip rotated positions - examination of electromyography during supine patella setting in a physically unimpaired person- [in Japanese]. J Hokkaido Phys Ther Assoc 2004;21:42-46.

18. Yoshio M, Murakami G, Sato T, Sato S, Noriyasu S: The function of the psoas major muscle: passive kinetics and morphological studies using donated cadavers. J Orthop Sci 2002;7:199-207. PMID:11956980, DOI:10.1007/s007760200034
19. Okubo Y, Kaneoka K, Hasebe K, Matsunaga N, Hodges P: Analysis of psoas major activity during active straight leg raise by using fine-wire electromyography. The 42nd Annual Meeting of the International Society for the Study of the Lumbar Spine 2015:132.

20. Hirano K, Kinoshita K, Thida M, Kawai Y, Kamikubo T, Abo M: The function of the iliacus using magnetic resonance imaging (MRI) - based on anatomical findings in cadavers [in Japanese]. J Jpn Phys Ther Assoc 2010;37:356-363.

21. Jiroumaru T, Kurihara T, Isaka T: Measurement of muscle length-related electromyography activity of the hip flexor muscles to determine individual muscle contributions to the hip flexion torque. Springerplus 2014;3:624. PMID:25392794, DOI:10.1186/2193-18013-624

22. Ogaya S, Tateuchi H, Takashima S, Ichihashi N: Change of torque-generating capacity of hip muscles according to the hip joint angle: a mathematical model analysis [in Japanese]. J Jpn Phys Ther Assoc 2011;38:97-104.

23. Blemker SS, Delp SL: Three-dimensional representation of complex muscle architectures and geometries. Ann Biomed Eng 2005;33:661-673. PMID:15981866, DOI:10.1007/s10439-005-1433-7

24. Kendall FP, Provance PG, McCreary EK: Muscles: Testing and Function, with Posture and Pain, 5th Edn. Lippincott Williams \& Wilkins, Philadelphia, 2005.

25. Skyrme AD, Cahill DJ, Marsh HP, Ellis H: Psoas major and its controversial rotational action. Clin Anat 1999;12:264-265. PMID:10398385, DOI:10.1002/ (SICI)1098-2353(1999)12:4<264::AID-CA4>3.0.CO;2-L

26. Keagy RD, Brumlik J, Bergan JJ: Direct electromyography of the psoas major muscle in man. J Bone Joint Surgery Am 1966;48:1377-1382. PMID:5921792, DOI:10.2106/00004623-196648070-00011

27. Nonaka H, Nakajima M, Akiyama J, Neya T: Effect of hip external rotation on muscle activity of lower extremity in SLR [in Japanese]. J Jpn Phys Ther Assoc 2004;31(Supplement.2):122. 